Authors' reply: We thank Dr Rao for his comments. We are sorry that it is unclear to Dr Rao as to precisely how the location of infarcts influenced the quantification of vascular pathology in this study. We would refer him to the discussion of neuropathological prevalence of dementia subtypes in which we hoped it was clearly stated that "the increased prevalence of infarction is more likely to reflect the stress laid on recording the presence of all infarctions regardless of size or location". Thus, when assessing the validity of these criteria no heed was taken as to whether they were to be considered as strategic or otherwise. Periventricular white matter lesions were not a prominent feature of these cases.

That he finds the low sensitivity of the NINDS-AIREN criteria to be striking is, of course, one of the main points of the paper but one that has been shown elsewhere and referenced in the paper (Gold et al, 1997).

The concept of a "nosologically heterogeneous group of disease processes" is precisely the concept that is at fault in dementia research, and one which impedes progress into looking for common causation. Indeed, we can do no better than also quoting Prince, but from a later paper, "It is perhaps regrettable that the clinical distinction between VAD and $A D$ has become so strongly established in advance of clear population-based epidemiological evidence for the existence of distinct disorders with discrete aetiologies" (Prince, 1995).

Gold, G., Giannaleopoulos, P., Montes-Puixuo, C., et ol (I997) Sensitivity and specificity of newly proposed clinical criteria for possible vascular dementia. Neurology. $49,690-694$.

Prince, M. (1995) Vascular risk factors and atherosclerosis as risk factors for cognitive decline and dementia. fournol of Psychosomotic Reseorch, 39. $525-530$.

c. Holmes University of Southampton, Thornhill Research Unit, Moorgreen Hospital, Southampton SO 30 3JB

N. Cairns Brain Bank Research Laboratory, Department of Neuropathology. Institute of Psychiatry, London SE5 8AF

\section{Neurodevelopmental delay in the severely ill}

Sir: Sigurdsson et al (1999) conclude that subjects with severe early-onset mood disorders have a lower IQ than those with mild or moderate forms of juvenile-onset affective illness, and present this finding as evidence supporting their hypothesis of neurodevelopmental delay in the severely ill group. We argue that their data do not support this conclusion.

Their study shows that a greater percentage of cases of severe early-onset affective disorder have an IQ below 85 compared with a control group of equally early-onset but milder, non-psychotic depression. For both cases and controls, Wechsler Intelligence Scale for Children (WISC (Wechsler, 1949), or its revision, WISC-R (Wechsler, 1974)) IQ data are available for only a minority of subjects $(42 \%)$. For the majority, the IQ estimate was taken from the original clinical assessment that took account of "all the available information". This is the non-standardised impression of various clinicians over an 18-year period. The authors present no correlation between measured $\mathrm{IQ}$ and estimated IQ, despite these data being available. There is, therefore, no measure of the validity of the estimated scores. Indeed, the authors note that low intelligence and learning difficulties were mentioned infrequently in referral letters and assessment summaries, which suggests that finding an IQ of less than 85 was unexpected in some of the subjects in which it was found. This also raises doubt about the validity of the estimated IQs. Further analysis of IQ is based only on the limited sample in whom formal WISC IQs are available. There is no attempt to assess whether this limited sample is representative of the cases and controls as a whole.

The authors give no clear account of the timing of IQ assessment, but imply that it was carried out during the index episode. This is a major potential confounder as IQ may be influenced by ongoing symptoms (for example, Sackeim et al, 1992); lower IQs in the more severely ill group could be a consequence of illness rather than an antecedent. To test the hypothesis a measurement of premorbid IQ is essential.

The assumption that IQ acts as a proxy measure for neurodevelopmental impairment can be challenged. Performance on intelligence tests is influenced by heredity, antenatal and infant nutrition (Lucas et al, 1992), various socio-economic factors (Elliott, 1988), gender (Hedges \& Nowell, 1995) and possibly ethnicity (Elliott, 1988). The cases and controls differed significantly in their parental country of origin, a measure of ethnicity, and the gender differences almost reached statistical significance.
Sigurdsson et al (1999) conclude that neurodevelopmental antecedents are overrepresented in severe early-onset mood disorder and that this is supported by contemporaneous IQ data. We argue that such use of incomplete measures of IQ is invalid. The use of unsatisfactory data to support their conclusions is unsafe.

Elliot, R. (1988) Tests, abilities, race and conflict. Intelligence, 12, 333-350.

Hedzes, L.V. \& Nowell, A. (1995) Sex differences in mental test scores, variability, and numbers of highscoring individuals. Science, $269,41-45$.

Lucas, A., Mortey R., Cole, T. J., et al (I992) Breast milk and subsequent intelligence quotient in children born preterm. Lancet, 339, 261-264.

Saclodim, H. A., Froeman, J., McEhiney M., ot of (IV92) Effects of major depression on estimate of intelligence. Journal of Clinical and Experimental Neuropsychology, 14, 268-288.

Sizurdseon, E., Fombonme, E. Syyal, K., et ol (19\%) Neurodevelopmental antecedents of early-onset bipolar affective disorder. British fournal of Psychiatry 174, 121-127

Wochaler, D. (1949) Manuol for the Wechsler Intelligence Scale for Children. New York: Psychological Corporation.

- (1974) Wechsler Intelligence Scale for Children Revised. New York: Psychological Corporation.

P. McConville, N. Walker University of Aberdeen. Department of Mental Health, Clinical Research Centre, Royal Cornhill Hospital, Cornhill Road, Aberdeen AB25 2ZH

Authors'reply: We are glad to be able to report in more detail the IQ findings from our study while responding to the points raised above. Three main issues need to be addressed. First, whether not having IQ test scores for all subjects (available for $42 \%$ of cases and $42 \%$ of controls) made it unsafe to use these scores to validate our main findings; second, the interpretation of the differences observed in IQ scores between the groups; and finally, whether it is appropriate to use IQ test scores as a proxy measure of neurodevelopmental antecedents in our sample.

With regard to the first issue, cases and controls for whom IQ test scores were available did not differ significantly from other cases and controls, respectively, with regard to gender, social class or parental country of birth. They were, however, slightly younger, on average, at the onset of the index episode. The magnitude of the age difference was similar but reached statistical significance only for the cases (cases $13.6 v .15 .0$ years, $P=0.019$; controls 13.8 v. 14.6 years, $P=0.23$, Student's $t$-test). Although the 